\title{
Características de la pubertad de escolares de sexo masculino de diferentes niveles socioeconómicos de la Región Metropolitana de Chile
}

\author{
Dr. Santiago Muzzo B.; Dra. Raquel Burrows A,; T,M. Laura Leiva B.; Sr. Abraham Zvaighaft F. ${ }^{1}$ \\ Characteristics puberty school age Chilean males \\ belonging to different socioeconomic levels
}

\begin{abstract}
The characteristies of puberal development were studied, in 2,535 school age males representative of high, medium and low socjoeconomic level (SEL) boys of the Metropolitan Region (Santiago, Chile). There was a wide range of ages at initiation and ending of genital and pubic hair development, wider at the lower SEL. The average age of initiation of genital development was lower in boys belonging to medium and low SEL (135.8 $\pm 14.9: 135.0 \pm$ 17.0 and $136.8 \pm 20.4$ months sespectively) compated with high SEL (141.9 \pm 14.9 months of age). The age at initiation of pubic hair was higher in school age boys belonging to low SEL (147.4 \pm 20.2 months) compared with the other groups (143.9 \pm 13.5 high; $141.7 \pm 15.8$ medjun and $142.5 \pm 15.3$ jn low $\$ E L)$. Farlier age at initial genital development than pubic hair development was found in subjects belonging to medium and low SEL. Among boys from high SEL there where were not such differences between this two last puberal events. Possible environmental and genetic factors involved in this findings are discussed.
\end{abstract}

(Key words: puberty, Chilean males, socioeconomic levels.)

La pubertad es el período de la adolescencia durante el cual se producen los cambios físicos, por acciones hormonales, que conducen a la madurez sexual y capacidad reproductiva del indiviđuo'.

En el desarrollo puberal influyen factores genéticos y ambientales ${ }^{2}$. Entre los factores ambientales, el estado de salud, la calidad de la nutrición, el nivel socioeconómico (NSE), la actividad física y las caracteristicas biogeográ ficas juegan un rol importante ${ }^{3-8}$. Esto determina diferencias en la edad de inicio y en la velocidad con que progresan los eventos de la pubertad. Asi, en adolescentes ingleses es posible encontrar todos los estadios del desarrollo puberal entre los 12 y 13 años de edad ${ }^{7}$.

La importancia de los factores genéticos y ambientales en la determinación de estas diferencias no está completamente dilucidada.

1. Lnidad de Endocrinología Infantil. Instituto de Nutrición y Tecnología de los Alimentos, Unjversidad de Chile.

- Proycato financiado parcialmente por FONDECYT $(94 / 85)$.
Varios estudios han demostrado que en mujeres existe un promedio de edad mayor de inicio de la pubertad y de presentación de la menarquia en las adolescentes de niveles socioeconómicos más bajos, con una tendencia secular a una menor edad de presentación de la menarquia. Sin embargo, también se ha observado que existen diferencias raciales que se sobreponen a las in. fluencias ambientales? $9-11$.

Si bien en varones existen pocos estudios al respecto, se ha demostrado la influencia de la nutrición en la edad de presentación de la pubertad. Se ha descrito un promedio de edad de estirón puberal mayor en adolescentes asiáticos y latinoamericanos ${ }^{12}$ con menor ingesta calórica. En adolescentes chilenos de niveles socioeconómicos medios se ha descrito un adelanto en la edad de estirón puberal en el varón chileno at compararlo con el europeo ${ }^{13,15}$.

Tomando en cuenta los factores mencionados, cada país debería contar con sus propios patrones de normalidad del desarrollo puberal, ya que no se puede extrapolar la cronología de estos eventos a diferentes poblaciones; incluso a una misma población en épocas o circunstan- 
cias ambientales diferentes. Es necesario estudiar cada población separadamente $\mathrm{y}$ reexaminarlas periódicamente. Nos interesó estudiar en escolares de sexo masculino de Santiago las características del desarrollo puberal y la influencia del NSE sobre este importante evento del desarrollo del adolescente.

\section{MATERIAL Y METODO}

Se efectuó un corte transversal de 2.535 varones en una mucstra representativa de escolares de 8 a 16 años de la Región Metropolitana de Chile. Para esto se obtuvo información del Ministerio de Educación sobre la distribución de los escolares en las edades antedichas, por tipo de colegio (fiscal, particular subvencionado y particulares) y por curso; y la muestra se obtuvo en iguales porcentajes.

El nivel socioeconómico (NSE) se evaluó por la escala de Graffar modificada ${ }^{16}$ que considera escolaridad $y$ actividad del jefe de hogar y calidad de la vivienda y los califica en una escala del 1 al 6 , donde 1 es el nivel más alto y 6 el más bajo. El grado de desarrollo puberal se evaluó según los estadios de Tanner que considera vello púbico y desarrollo genital en el varón 6 . Se utilizó un orquidónetro de Prader y un pie de metro (Vernier) para la medición del volumen testicular, diámetro y longitud peneana, respectivamente. El análisis estadístico de los resultados fue realizado aplicando un análisis de varianza (Prueba $F$ ), $t$ de Student, chi cuadrado y los promedios de edades se realizaron de acuerdo al método de próbito.

\section{RESULTADOS}

Observamos que entre los 12 y 14 años 11 meses existen escolares en todas las etapas de desarrollo de vello púbico. A los 8 años de edad hubo un 2,6\% de escolares con inicio de vello púbico $y$ entre los 14 años 6 meses y los 14 años 11 meses aun existió un $5,8 \%$ de varones sin vello púbico. Hubo un escolar de 11 años y otro de 12 años con desarrollo completo del vello púbico. Después đe los 12 affos 11 meses predominan los escolares con desarrollo del vello púbico IlI o más.

Observamos la siguiente distribución de estos escolares en las diferentes etapas del desarrollo genital. A los 8 años de edad hay un $4,5 \%$ de los escolares que ya inició el desarrollo genital y entre los 14 años y 6 meses y los 14 años y 11 meses existe un 2,6* que aún no empieza. Entre $\operatorname{los} 11$ y $\operatorname{los} 14$ años y 11 meses hay varones en todas las etapas del desarrollo genital, a los 12 años de edad más del $70 \%$ inició ya este desarrollo.
Se analizó la distribución porcentual por edad de los escolares que ya están en la etapa inicial del desarrollo genital y vello púbico (G2 y $\mathrm{P} 2$, respectivamente). Para ambos eventos, el mayor porcentaje de escolares se acumula entre los 12 y 14 años, con un rango que va desde los 8 hasta los 15 affos. El porcentaje máximo de varunes en etapa inicial de desarrollo genital y púbico está entre los 12 y 12 años 11 meses $(42,2 \%$ y $38,7 \%$, respectivamente). También puede verse que en los escolares menores de 12 años hay un mayor porcentaje de niños con inicio de desarrollo genital que de púbico, en los distintos grupos etarios analizados.

La tabla 1 muestra los rangos de edad para etapas iniciales y finales de desanollo genital y púbico, por nivel socioeconómico. En todos los niveles socioeconómicos se observó un amplio rango de edad tanto en las etapas iniciales (P2 y G2) como en las finales (P5 y G5) del desarrollo puberal. Estos rangos fueron menores en los escolares de nivel alto (72 meses para P2, 60 para G2 y 31 para P5 y G5) al compararlos con los de nivel bajo (101 meses para $\mathrm{P} 2,84$ para $\mathrm{G} 2$ y 47 para P5 y G5).

Al analizar la edad promedio de las diferentes etapas del desarrollo genital (calculada por el método de próbitos) se observaron diferencias por nivel socioeconómico (tabla 2). Los escolares de NSE alto $(1+2)$ presentaron un promedio de edad de inicio del desarrollo genital (141,91 $\pm 14,97$ meses) significativamente mayor (p $<0,01)$ a la de los niveles medios $(3$ y 4$)$ $(135,69 \pm 17,56$ y $135,03 \pm 17,04$ meses $)$ y bajo $(5+6)(136,84 \pm 20,48$ meses $)$. Sin embargo, en las etapas posteriores ( $\mathrm{G} 3$ y $\mathrm{G} 4$ ), los escolares de nivel bajo presentan un mayor promedio de edad $(172,87 \pm 13,96$ meses $)$ al compararlos con los de niveles medio, medio bajo y alto $(166,17 \pm$ 16,$26 ; 169,00 \pm 15,09$ y $166,29 \pm 12,68$, respectivamente) $(\beta<0,01)$.

Al observar el promedio de edad de estos escolares en las diferentes etapas del vello púbico (tabla 3), también existen diferencias por NSE. En las tres etapas analizadas (P2, P3 y P4) el promedio de edad fue significativamente mayor ( $\mathrm{p}<0,01)$ en los escolares de NSE bajo $(147,41 \pm 20,25 ; 166,15 \pm 14,28$ y $175,10 \pm$ 14,39 , respectivamente) al compararlos con el resto de los niveles en las etapas 2 y 3 y con el nivel medio $y$ alto en la etapa 4 . 
Tabla 1

Rangos de edad (meses) para etapas iniciales y finales de desarrollo genital y vello púbico en varones de 8 a 16 años según NSE

\begin{tabular}{lcccr}
\hline $\begin{array}{l}\text { Litapa de } \\
\text { desarrollo } \\
\text { puberal }\end{array}$ & \multicolumn{3}{c}{ Nivel socioeconómico (Graffar) } \\
\cline { 2 - 5 } & $1+2$ (Alto) & 3 (Medio) & 4 (Medio bajo) & $5+6$ (Bajo) \\
\hline P2 & $108-180$ & $108-186$ & $99-189$ & $97-188$ \\
G2 & $113-173$ & $101-186$ & $99-189$ & $97-181$ \\
PS & $159-190$ & $132-199$ & $145-203$ & $156-203$ \\
G5 & $159-190$ & $132-199$ & $145-203$ & $156-203$ \\
\hline
\end{tabular}

P2: Estadio It de vello púbico.

G2: Estadio II de desartollo genital.

P5: Estatio $V$ de vello púbico.

G5: Estadio $\mathrm{V}$ de desarrollo genital.

NSE: Nivel socioeconómico.

Tabla 2

Edad promedio (meses) en diferentes etapas de desarrollo genital en varones de 8 a 16 años por nivel socioeconómico

\begin{tabular}{|c|c|c|c|c|c|c|c|}
\hline \multirow{2}{*}{$\begin{array}{l}\text { Etapa de } \\
\text { Taunes }\end{array}$} & \multicolumn{7}{|c|}{ Nivel socioeconómico (Graffar) } \\
\hline & $1+2(A)$ & $3(\mathrm{~B})$ & $4(\mathrm{C})$ & $5+6(D)$ & $\mathrm{p}^{\mathrm{b}}$ & $\mathrm{p}^{\mathrm{c}}$ & \\
\hline G2 & ${ }_{(239)}^{141,91 \pm 14,97 a}$ & $\begin{array}{c}135,84 \pm 17,56 \\
(375)\end{array}$ & $\frac{135.03 \pm 17.04}{(S 85)}$ & ${ }_{(234)}^{136,84 \pm 20,48}$ & 0,01 & $\begin{array}{l}\mathrm{A} / \mathrm{D} \\
\mathrm{A} / \mathrm{C} \\
\mathrm{A} / \mathrm{B}\end{array}$ & $\begin{array}{l}0,00 \\
0,00 \\
0,00\end{array}$ \\
\hline G3 & $154,23 \underset{(88)}{ \pm} 11,72$ & $\frac{151,67 \pm 15,07}{(289)}$ & ${ }_{(415)}^{153,08} \pm 16,91$ & $159,41 \pm 16,49$ & 0,01 & $\begin{array}{l}\mathrm{A} / \mathrm{D} \\
\mathrm{B} / \mathrm{D} \\
\mathrm{C} / \mathrm{D}\end{array}$ & $\begin{array}{l}0,01 \\
0,00 \\
0,00\end{array}$ \\
\hline G4 & $166,29 \underset{(51)}{ \pm} 12,68$ & $166,17 \underset{(176)}{ \pm} 16,26$ & ${ }_{(235)}^{169,00 \pm 15,09}$ & $172,87 \underset{(74)}{ \pm} 13,96$ & 0,01 & $\begin{array}{l}A / D \\
B / D \\
C^{\prime} D\end{array}$ & $\begin{array}{l}0,01 \\
0,00 \\
0,05\end{array}$ \\
\hline
\end{tabular}

$\mathrm{a}=\overline{\mathrm{X}}(\mathrm{DE})$ (número de casos) por método đe próbitos.

$\mathrm{b}=$ significancia según prueba $\mathrm{F}$.

$c=$ significancia según t de Student.

Tabla 3

Edad promedio (meses) en diferentes etapas de vello púbico en varones đe diferentes niveles socioeconómicos

\begin{tabular}{|c|c|c|c|c|c|c|c|}
\hline \multirow{2}{*}{$\begin{array}{l}\text { Ettapa de } \\
\text { Tanuer }\end{array}$} & \multicolumn{7}{|c|}{ Nivel socioeconómico (Graftar) } \\
\hline & $1+2(\mathrm{~A})$ & $3(\mathbf{B})$ & $4(C)$ & $5+6(0)$ & $\mathrm{p}^{\mathrm{b}}$ & $\mathrm{p}^{\mathrm{c}}$ & \\
\hline $\mathrm{P} 2$ & $\begin{array}{c}143,98 \pm 13,55^{a} \\
(118)\end{array}$ & $141,71 \underset{(346)}{ \pm} 15,85$ & $c_{(537)}^{142,57 \pm 15,39}$ & $\begin{array}{c}147,41 \pm 20,25 \\
(192)\end{array}$ & 0,01 & $\begin{array}{l}A / D \\
B / D \\
C / D\end{array}$ & $\begin{array}{l}0,05 \\
0,001 \\
0,005\end{array}$ \\
\hline $\mathrm{P3}$ & $156,71 \underset{(81)}{ \pm} 13,74$ & ${ }_{(259)}^{155,35 \pm 14,71}$ & $\underset{(369)}{158,51 \pm 12,79}$ & ${ }_{(106)}^{166,15 \pm 14,28}$ & 0,01 & $\begin{array}{l}\mathrm{A} / \mathrm{D} \\
\mathrm{B} / \mathrm{D} \\
\mathrm{C}^{\prime} \mathrm{D}\end{array}$ & $\begin{array}{l}0,001 \\
0,001 \\
0,001\end{array}$ \\
\hline $\mathrm{P} 4$ & $167,37 \pm 13,61$ & ${ }_{(162)}^{168,04 \pm 16,36}$ & ${ }_{(217)}^{171,24 \pm 15,66}$ & $175,10 \underset{(67)}{ \pm} 14,39$ & 0,01 & $\begin{array}{l}A / D \\
B / D\end{array}$ & $\begin{array}{l}0,005 \\
0,005\end{array}$ \\
\hline
\end{tabular}

$a=\bar{X}$ (DE) (número de casos) por método de próbitos.

$b=$ significancia segín prueba $\mathrm{F}$.

c - significancia según t de Student. 
Al comparar el promedio de edad de los escolares en la etapa inicial del desarrollo genital con la del vello púbico, también hay diferencias por NSE (tabla 4). El promedio de edad es significa. tivamente menor para el inicio del desarrollo genital que para el púbico en los escolates de niveles medios y bajos $(\mathrm{p}<0,0005)$, en tanto que fue similar en los escolares del nivel alto. Las tablas 5 y 6 muestran los promedios de longitud y diámetro peniano en escolares de 13 a 16 años que se encuentran en etapa P2 y P4. No se encontraron diferencias por NSE. El mismo análisis se hizo con el volumen testicular (tabla 7) y no se encontró diferencias por NSE. Sin embargo, en P4 hubo un porcentaje significativamente mayor de escolares con volumen testicu. lar sobre los $15 \mathrm{cc}$ a medida que se deteriora el NSE $(\mathrm{p}<0,05)$.

Tabla 4

Edad promedio (meses) de inicio de desarrollo genital y vello púbico en varanes pot nivel socioeconómico

\begin{tabular}{|c|c|c|c|}
\hline \multirow{2}{*}{$\begin{array}{l}\text { Nivel socioeconómico } \\
\text { (Graffar) }\end{array}$} & \multicolumn{2}{|c|}{ Grado de desarrollo puberal (Tanner) } & \multirow[t]{2}{*}{$\mathbf{p}^{b}$} \\
\hline & $\mathrm{G} 2$ & $\mathrm{P} 2$ & \\
\hline $1+2$ & $141,91 \underset{(239)}{ \pm} 14,97 \mathrm{a}$ & ${ }_{(118)}^{143,98 \pm 13,55}$ & N.S. \\
\hline 3 & ${ }_{(375)}^{135,84}$ & $141,71 \pm 1 \pm 15,85$ & $<0,0005$ \\
\hline 4 & $135,03 \pm 17 \pm 17,04$ & $\begin{array}{c}142,57 \pm 15,39 \\
(537)\end{array}$ & $<0,0005$ \\
\hline $5+6$ & $\begin{array}{c}136,84 \pm 20,48 \\
(234)\end{array}$ & $147,41 \pm 20,25$ & $<0,0005$ \\
\hline
\end{tabular}

$a=\bar{X}$ (DE) (numero de casos) por método de próbitos.

$\mathrm{b}=$ significancia según $\mathrm{t}$ de Student.

Tabla 5

Longitud peniana (mm) en escolares de 13 a 16 años en etapas II y IV de vello púbico por nivel socioeconómico

\begin{tabular}{|c|c|c|c|c|c|}
\hline \multirow{2}{*}{$\begin{array}{l}\text { Estadio de } \\
\text { vello púbico }\end{array}$} & \multicolumn{5}{|c|}{ Nivel socioeconómico (Graffar) } \\
\hline & $1+2(A)$ & $3(\mathrm{~B})$ & $4(C)$ & $5+6(\mathrm{D})$ & $\mathrm{p}^{\mathrm{b}}$ \\
\hline II & $43,3 \underset{(16)}{ \pm} 11,0^{a}$ & $36,0 \pm 9,0$ & $38,0 \underset{(48)}{ \pm} 10,0$ & $38,0 \pm 9,0$ & N.S \\
\hline $\mathrm{JV}$ & $57,0 \underset{(26)}{ \pm} 6,8$ & $59,0 \pm 9,5$ & $59,0 \underset{(122)}{ \pm} 9,1$ & $58,8 \underset{(24)}{ \pm} 1,2$ & N.S. \\
\hline
\end{tabular}

$\mathrm{a}=\overrightarrow{\mathrm{X}}$ (DE) (número de casos).

$\mathrm{b}=$ significancia según prueba $\mathrm{F}$. 
Tabla 6

Diánetro peniano (mm) de escolates de 13 a 16 arios en etapas II y IV de vello púbico por nivel socioeconómico

\begin{tabular}{|c|c|c|c|c|c|}
\hline \multirow{2}{*}{$\begin{array}{l}\text { Estadio de } \\
\text { vello púbico }\end{array}$} & \multicolumn{5}{|c|}{ Nivel socioeconómico (Graffar) } \\
\hline & $1+2(A)$ & 3 (B) & $4(C)$ & $5+6$ (D) & $\mathrm{p}^{\mathrm{b}}$ \\
\hline$\amalg$ & $\begin{array}{c}23,88 \pm 4,21^{a} \\
(16)\end{array}$ & $22,81 \pm 3,75$ & $21,69 \pm 3,74$ & $21,43 \pm 3,23$ & A.S. \\
\hline IV & $28,71 \pm 2,93$ & $\begin{array}{c}29,28 \pm 2,87 \\
(87)\end{array}$ & $\begin{array}{c}29,30 \pm 3,47 \\
(124)\end{array}$ & $\begin{array}{c}29,75 \pm 2,94 \\
(24)\end{array}$ & N.S. \\
\hline
\end{tabular}

$a=\overline{\mathbf{X}}$ (DE) (número de casos).

b a significancia scgún prueba $\mathrm{F}$.

Tabla 7

Volumen testicular $\left(\mathrm{cm}^{3}\right)$ en escolares de 13 a 16 años en etapa Il y IV de vello púbico por nivel socioeconómico

\begin{tabular}{|c|c|c|c|c|c|c|c|c|c|}
\hline \multirow{3}{*}{$\begin{array}{l}\text { Vello } \\
\text { púbico }\end{array}$} & \multirow{3}{*}{$\begin{array}{c}\text { Volumen } \\
\text { testicular } \\
\left(\mathrm{cm}^{3}\right)\end{array}$} & \multicolumn{8}{|c|}{ Nivel socioeconómico (Graffar) } \\
\hline & & \multicolumn{2}{|c|}{$1+2$} & \multicolumn{2}{|c|}{3} & \multicolumn{2}{|c|}{4} & \multicolumn{2}{|c|}{$5+6$} \\
\hline & & $\overline{\mathrm{n}}$ & $\%$ & $\mathrm{n}$ & 8 & $\mathfrak{n}$ & $\%$ & $\pi$ & $\%$ \\
\hline \multirow{3}{*}{ II } & $<10$ & 9 & 56,3 & 21 & 72,4 & 40 & 85,1 & 19 & 86,4 \\
\hline & $\geqslant 10$ & $?$ & 43,7 & 8 & 27,6 & 7 & 14,9 & 3 & 13,6 \\
\hline & TOTAL & 16 & 100,0 & 29 & 100,0 & 47 & 100,0 & 22 & 100,0 \\
\hline
\end{tabular}

$P=$ N.S.

\begin{tabular}{llllllllll}
\hline IV & 11 & 73,3 & 34 & 40,0 & 48 & 39,7 & 6 & 25,0 \\
$\geqslant 15$ & 4 & 26,7 & 51 & 60,0 & 73 & 60,3 & 18 & 75,0 \\
\cline { 2 - 9 } & TOTAL & 15 & 100,0 & 85 & 100,0 & 121 & 100,0 & 24 & 100,0 \\
\hline
\end{tabular}

\section{DISCUSION}

Se efectuó un corte transversal en una muestra de 2.535 varones, entre 8 y 16 afos, representativa de escolares de la Región Metropolitana, para investigar algunas características del desarrollo puberal. Entre los 11 y los 14 años de edad se encontraron varones en todas las etapas del desarrollo genital y púbico con un gran rango de edad en el inicio y término del desarrollo puberal. Este rango fue mayor en escolares de nivel bajo al compararlos con los de nivel alto. Este amplio rango también fue encontrado en mujeres representativas de escolares de sexo femenino de la misma región ${ }^{17}$ y ha sido descrito en escolares chilenos de nivel medio y europeos ${ }^{6-7,13-14}$; este hecho debe ser considerado para un mejor enfoque y estudio en adolescentes con pubertades tempranas y tardias.

Se encontró un desfase entre la edad de apari. ción del desarrollo genital y púbico en escolares de NSE medios y bajos, hecho que no se observó en los de nivel alto. Esto fue dado por una menor edad de inicio del desarrollo genital en los escola- 
res de niveles medios y bajos. Sin embargo, los escolares de nivel bajo alcanzaron las edades posteriores del desarrollo genital y todas las del vello púbico a edades mayores, por lo que fue interesante analizar si existían mayores volúmenes testiculares en escolares de clase baja, al compararlos con los de nivel alto, que hicieran sospechar por el tamaño testicular un inicio más temprano del desarrollo genital. -El estudio de volumen testicular en etapa $\mathrm{P} 2$ (que supone una mayor edad para los escolares de nivel bajo) no apoyó esta hipótesis. Por otro lado hemos encontrado un inicio más temprano de la pubertad en mujeres de clase media al compararlas con las de clase alta y algo similar ha sido descrito por otros investigadores chilenos, quienes encontraron una pubertad más temprana en adolescentes de clase media de ambos sexos al compararlos con europeos ${ }^{i 5}$.

Seria interesante investigar factores racjales que pudieran explicar estos hechos, ya que en escolares de NSE alto el porcentaje de ancestros europeos (exclujdo el español), evaluado a través de los 4 apellidos, fue significativamente mayor que en los otros niveles en los que predominan los apellidos españoles y aumentan los apellidos aborigenes a medida que disminuye el NSE ${ }^{18}$. Es poco probable que factores ambientales de tipo nutricional estén acelerando la pubertad en los escolares de niveles más bajos, ya que no hemos encontrado diferencias con respecto a estado nutricional por NSE y el estudio de composición corporal reveló que ésta fue más adecuada a mayor NSE ${ }^{19}$.

\section{RESUMEN}

Se analizaron algunas caracteristicas del desarrollo puberal en 2.535 varones, de 8 a 16 años, representativos de escolares de la Región Metropolitana. Se investigaron las diferencias de acuerdo al NSE. Se encontró un amplio rango en la edad de inicio y en la de término del desarrollo genital y púbico, el cual fue mayor en escolares de nivel bajo, al compararlos con los de nive] alto. Entre $\operatorname{los} 12$ y 14 años hubo escolares en todas las etapas del desarrollo genital y púbico. Se encontraron diferencias significativas por NSE, con respecto a la edad promedio del inicio del desarrollo genital y púbico. El promedio de edad de inicio del desarrollo genital fue menor en escolares de niveles medios y bajos $(135,84 \pm$
14,$97 ; 135,03 \pm 17,04$ y $136,84 \pm 20,48$ meses, respectivamente) que en los de nivel alto (141,91 $\pm 14,97$ meses); en tanto que la edad de inicio del vello púbico fue mayor en escolares de nivel bajo $(147,41 \pm 20,25$ meses $)$ al compararlos con el resto $(143,98 \pm 13,55$ en nivel alto, $141,71 \pm 15,85$ en nivel medio y $142,57 \pm 15,39$ en nivel medio bajo). Hubo diferencias por NSE al comparar edad de inicio de ambos eventos puberales (desarrollo genital y púbico), observándose una partida más temprana del desarrollo genital que del púbico en los escolares de niveles medio y bajo, en tanto que los de nivel alto parten simultáneamente con ambos eventos. Se plantea la posible influencia de factores raciales para explicar estas diferencias.

\section{REFERENCIAS}

1. Ducharme J.R., Calls R.: Pubertal development: normal precocius and delayed. Clin Endocrinol Metab 1982;11:57-87.

2. Shenkez R.I., Nusshaum M, and Kaplan E. Delayed puberty and short stature in adolescents. Pediatr Ann 1978; 7:56-66.

3. Kennedy G.C. and Mitra J.: Bady weight and food intake as initiating factors for puberty in the rat. J Physiol 1963; 166: 405-414.

4. Frisch R.E.: Body fact, puberty and fertility. Biol Rev 1984; 59 : 161-188.

5. Zachurias $l$., Band $W$., Wurtman $R . J$. A prospective study of sexual development and growth in American girls: the statistics of menarche. Obstet Gynecol 1976:31: 325-337.

6. Frish $R, E$, Critical weight at menarche, initiation of the adolescent growth spurt, and control of puberty. En Grumbach, Grove and Mayer Control of the onset of puberty. Willy Ed. New York 1974: 403-423.

7. Marshall W.A. and Tanner J.M.: Variations in the Pattern of pubertal changes in boys. Afch Dis Child $1970 ; 45: 13-23$.

8. Belmacker $E$ : : Sexual maturation of Jerusalem schoolgirls and its a association with socio-economic factor and ethric group. Ann Hum Biol 1982; 9: 321-328.

9. Patri M.A., Valenzizela C., Morales C.I., Saavedra Z.I.. Figueroa O.L.: Estudio de la edad de la menarquia en niñas escolares de la enseñanza finscal del sector norte de Santiago. Cuad Med Soc 1980; 21: $12-20$.

10. López C.M., Tobar E.G., Farid C.N., Landaeto J.M., Méndez C.H.: Estudios comparados de la cstatura y edad de la menarquia según estrato socjoeconómico en Venezuela. Arch Latinoam Nutr $1981 ; 31: 740-757$.

11. Rona R.: Secular tient of pubertal development in Chile. J Hum Evol 1975:4: 251.257. 
12. Frisch R.E., Revelle R.: Variation in body weights and the age of the adolescent growth spurt among Latin American and Asian population in relation to caloric supplies. Hum Biol $1969 ; 41$ : 85-212.

13. Avendaño B.A., Patrí M.A., Valenzuela C.: Antropometría de escolares fiscales del Area Metropolitana Norte de Santiago. 3ra. parle. Maduración sexual. Cuad Med Soc 1975; 16: 17-24.

14. Valenzuela C., Avendaño B.A.: Antropornetría $y$ maduración sexual de escolares de un área de Santiago de Chile. Bol of Sanit Panam 1969; 87: 113.131 .

15. Valenzuela $C .:$ Adelanto de la pubertad de escolares chilenos respecto a escolares europeos medido por la velocidad de crecimiento estatural en un estudio transversal. Pediatría, Santiago, Chile 1977; 20: 74-78.
16. Alvarez M., Wurgaft F., Salazay M.: Mediciones del nivel socioeconómico bajo urbano en familias con lactantes desnutridos. Arch Latinoam Nutr 1982:21: 650 .

17. Burrows R., Leiva L., Matricci A., Zvoighaft A., Muzzo S.: Características de la pubertad de escolases chilenos de sexo femeníno de la Región Metropolitana. Rev Chil Pediatr 1988: 59: 21-25.

18. Muzzo S., Burrow's R., Leive L., Zvaighaft A., Alvarez M.L.: Caracteristicas biológicas, psicológicas y socioculturales del adolescente chileno de diferentes NSE. Informe final Proyecto 93/85. FONDECYT, 1986.

19. Leiva L, Mauricci A., Burrow's R., Zvaighaft A., Kauschartski A., Muzzo S.: Estado nutricional durante el desarrollo puberal de escolares del Area Metropolitana de Chile. Rev Chil Nutr 1986 14: $206-214$. 